\title{
REGULAR SPACES OF SMALL EXTENT ARE $\omega$-RESOLVABLE
}

\author{
ISTVÁN JUHÁSZ, LAJOS SOUKUP, AND ZOLTÁN SZENTMIKLÓSSY
}

\begin{abstract}
We improve some results of Pavlov and of Filatova, respectively, concerning a problem of Malychin by showing that every regular space $X$ that satisfies $\Delta(X)>\mathrm{e}(X)$ is $\omega$-resolvable. Here $\Delta(X)$, the dispersion character of $X$, is the smallest size of a non-empty open set in $X$ and $\mathrm{e}(X)$, the extent of $X$, is the supremum of the sizes of all closed-and-discrete subsets of $X$. In particular, regular Lindelöf spaces of uncountable dispersion character are $\omega$-resolvable.

We also prove that any regular Lindelöf space $X$ with $|X|=$ $\Delta(X)=\omega_{1}$ is even $\omega_{1}$-resolvable. The question if regular Lindelöf spaces of uncountable dispersion character are maximally resolvable remains wide open.
\end{abstract}

\section{INTRODUCTION}

We start by recalling a few basic definitions and facts concerning resolvability. A topological space $X$ is said to be $\lambda$-resolvable ( $\lambda$ a cardinal) if $X$ contains $\lambda$ many mutually disjoint dense subsets. A natural upper bound on the resolvability of $X$ is

$$
\Delta(X)=\min \{|G|: G \text { is non-empty open in } X\},
$$

called the dispersion character of $X$. So, $X$ is said to be maximally resolvable if it is $\Delta(X)$-resolvable. The expectation is that "nice" spaces should be maximally resolvable, an expectation verified e.g. by the well-known facts that compact Hausdorff, or metric, or linearly ordered spaces are all maximally resolvable.

It is also well-known, however, that there is a countable regular (hence "nice") space with no isolated points that is irresolvable, i.e. not even 2-resolvable. Since countable spaces are (hereditarily) Lindelöf, this prompted Malychin to ask the following natural question in

Date: November 8, 2013.

2010 Mathematics Subject Classification. 54A35, 03E35, 54A25.

Key words and phrases. resolvable, Lindelöf, countable extent.

The research on and preparation of this paper was supported by OTKA grant no. K 83726. 
[6]: Is every regular Lindelöf space of uncountable dispersion character (at least 2-)resolvable? He also noted that the answer to this question is negative if regular is weakened to Hausdorff.

Pavlov in [7] proved the following very deep result that gave a partial affirmative answer to Malychin's question: If $X$ is any regular space satisfying $\Delta(X)>\mathrm{e}(X)^{+}$then $X$ is $\omega$-resolvable. (In fact, he only needed the following assumption on $X$ that we call $\pi$-regularity and is clearly weaker than regularity: The regular closed sets in $X$ form a $\pi$-network, i.e. every non-empty open set includes a non-empty regular closed set.)

We recall that the extent $\mathrm{e}(X)$ of $X$ is the supremum of the sizes of all closed-and-discrete subsets of $X$. Since Lindelöf spaces have countable extent, it followed that regular Lindelöf spaces of dispersion character $>\omega_{1}$ are $\omega$-resolvable. Thus only the case $\Delta(X)=\omega_{1}$ of Malychin's problem remained and that was settled by Filatova in [2]: Any regular Lindelöf space $X$ with $\Delta(X)=\omega_{1}$ is 2-resolvable. However, her method of proof did not seem to give even 3-resolvable, not to mention $\omega-$ resolvable as in Pavlov's result.

Our main result in this paper, theorem 3.1, improves Pavlov's above result by showing that the assumption $\Delta(X)>\mathrm{e}(X)^{+}$in it can be relaxed to $\Delta(X)>\mathrm{e}(X)$. This, of course, immediately implies that Filatova's 2-resolvable can also be improved to $\omega$-resolvable. We also think that the proof of our strengthening of Pavlov's result is significantly simpler than Pavlov's original proof, especially in the case when $\Delta(X)$ is singular.

We do not know, however, if a regular space $X$ satisfying $\Delta(X)>$ $\mathrm{e}(X)$ is always maximally resolvable, or even if regular Lindelöf spaces of uncountable dispersion character are maximally resolvable. This problem should be confronted with our result from [4] stating that any topological space $X$ satisfying $\Delta(X)>s(X)$ is maximally resolvable. Here $s(X)$, the spread of $X$, is the supremum of the sizes of all (relatively) discrete subsets of $X$.

Theorem 4.1 in this paper implies that, for any infinite cardinal $\kappa$, if all regular Lindelöf spaces of cardinality and dispersion character $\kappa^{+}$ are $\kappa$-resolvable then all such spaces are actually $\kappa^{+}$-resolvable as well. This then, together with theorem 3.1, implies that any regular Lindelöf space $X$ with $|X|=\Delta(X)=\omega_{1}$ is even $\omega_{1}$-resolvable, i.e. maximally resolvable. Considering that after Pavlov and before Filatova this was the unsolved "hard case" of Malychin's problem, for which even 2resolvability was unknown, it seems to be not unreasonable to raise the question if regular Lindelöf spaces of uncountable dispersion character are maximally resolvable. 


\section{Preliminary Results}

In this section we have collected some known and some new results that will play an essential role in the proof of our main results, theorems 3.1 and 4.1 .

First we fix a couple of important pieces of notation: For a topological space $X$, we denote by $\tau^{+}(X)$ the collection of all non-empty open sets in $X$ and by $\mathrm{RC}^{+}(X)$ the family of all non-empty regular closed subsets of $X$. As we have noted above, if $X$ is regular then $\mathrm{RC}^{+}(X)$ is a $\pi$-network in $X$.

We shall make very frequent use of the following simple but basic result.

Theorem 2.1 (Elkin, [1]). If $X$ is a topological space, $\kappa$ is any cardinal, and the family

$$
\mathfrak{R}_{\kappa}(X)=\{Z \subset X: Z \text { is } \kappa \text {-resolvable }\}
$$

is a $\pi$-network in $X$ then $X \in \mathfrak{R}_{\kappa}(X)$, i.e. $X$ is $\kappa$-resolvable.

Since for every $G \in \tau^{+}(X)$ there is $H \in \tau^{+}(X)$ such that $H \subset G$ and $|H|=\Delta(H)$, moreover then $R \in \mathrm{RC}^{+}(X)$ and $R \subset H$ imply $|R|=$ $\Delta(R)(=|H|)$, we obtain the following simple but useful corollary.

Corollary 2.2. Let $\mathcal{C}$ be a regular closed hereditary class of regular spaces, i.e. such that $X \in \mathcal{C}$ implies $\mathrm{RC}^{+}(X) \subset \mathcal{C}$. If every space $X \in \mathcal{C}$ with $|X|=\Delta(X)$ has a $\kappa$-resolvable subspace then every member of $\mathcal{C}$ is $\kappa$-resolvable.

In proving that certain spaces are $\omega$-resolvable, like in the proof of theorem 3.1, the following result comes naturally handy.

Theorem 2.3 (Illanes, [3]). If a topological space $X$ is $k$-resolvable for each $k<\omega$ then $X$ is $\omega$-resolvable.

Now we turn to formulating and proving some new results that will be needed later, in the proofs of our main theorems 3.1 and 4.1. They may turn out to be of independent interest.

First we fix some, rather standard, notation: If $A$ is any subset of a topological space then $A^{\prime}$ denotes the derived set of $A$, that is the set of all accumulation points of $A$, while we use $A^{\circ}$ to denote the set of all complete accumulation points of $A$.

The following rather technical result is new, although it owes its basic idea to Filatova's work in [2]. 
Lemma 2.4. Let $X$ be a regular space, $\kappa$ be a regular cardinal, and consider the family

$\mathcal{D}=\left\{D \in[X]^{\kappa}: D^{\prime}=D^{\circ}, D \cap D^{\circ}=\emptyset\right.$, and $\left.\forall E \in[D]^{\kappa}\left(E^{\circ} \neq \emptyset\right)\right\}$.

If $X$ has a dense subset $Y$ with $|Y| \leq \kappa$ such that for each point $y \in Y$ there is a set $D \in \mathcal{D}$ with $y \in D^{\circ}$ then $X$ is 2-resolvable.

Proof of the lemma. First, let us fix a $\kappa$-type enumeration of $Y$ (with repetitions permitted): $Y=\left\{y_{\alpha}: \alpha<\kappa\right\}$. We shall then, by induction on $\alpha<\kappa$, define $D_{\alpha} \in \mathcal{D}$ and $i_{\alpha} \in 2$ in such a way that, putting for $\alpha \leq \kappa$ and $i<2$

$$
E_{\alpha, i}=\bigcup\left\{D_{\beta}: \beta<\alpha \text { and } i_{\beta}=i\right\} \cup \bigcup\left\{D_{\beta}^{\circ}: \beta<\alpha \text { and } i_{\beta}=1-i\right\} \text {, }
$$

for any $\alpha \leq \kappa$ we have both

$$
E_{\alpha, 0} \cap E_{\alpha, 1}=\emptyset
$$

and

$$
\left\{y_{\beta}: \beta<\alpha\right\} \subset \overline{E_{\alpha, 0}} \cap \overline{E_{\alpha, 1}} \text {. }
$$

To start with, we pick $D_{0} \in \mathcal{D}$ with $y_{0} \in D_{0}^{\circ}$ and put $i_{0}=0$. Then (2.2) and (2.3) are trivially satisfied. Now, assume that $0<\alpha<\kappa$, moreover $\left\{D_{\beta}: \beta<\alpha\right\}$ and $\left\{i_{\beta}: \beta<\alpha\right\}$ have been defined and satisfy the inductive hypotheses (2.2) and (2.3).

We now distinguish three cases. First, if

$$
y_{\alpha} \in \overline{E_{\alpha, 0}} \cap \overline{E_{\alpha, 1}}
$$

then we may simply let $D_{\alpha}=D_{0}$ and $i_{\alpha}=i_{0}=0$. Clearly, then (2.2) and (2.3) will remain valid for $\alpha+1$.

Next, if

$$
y_{\alpha} \notin \overline{E_{\alpha, 0}} \cup \overline{E_{\alpha, 1}}
$$

then, using the regularity of $X$, we can pick an open neighbourhood $U$ of $y_{\alpha}$ for which $\bar{U} \cap \overline{\bigcup_{\beta<\alpha} D_{\beta}}=\emptyset$. Now choose $D \in \mathcal{D}$ with $y_{\alpha} \in D^{\circ}$ and set $D_{\alpha}=U \cap D$, moreover let $i_{\alpha}=0$. Again, it is easy to check that with these choices (2.2) and (2.3) remain valid.

If none of the above two alternatives hold then $y_{\alpha} \in \overline{E_{\alpha, 0}} \triangle \overline{E_{\alpha, 1}}$, i.e. there is $j \in 2$ such that $y_{\alpha} \in \overline{E_{\alpha, j}} \backslash \overline{E_{\alpha, 1-j}}$. Suppose e.g. that $j=0$, the case $j=1$ can be handled symmetrically.

We may then choose an open neighbourhood $U$ of $y_{\alpha}$ such that $\bar{U} \cap$ $\overline{E_{\alpha, 1}}=\emptyset$ and a set $D \in \mathcal{D}$ with $y_{\alpha} \in D^{\circ}$. For every $\beta<\alpha$ we have $\left|U \cap D_{\beta}\right|<\kappa$ : Indeed, if $i_{\beta}=0$ then $\left|U \cap D_{\beta}\right|=\kappa$ would imply

$$
\emptyset \neq\left(U \cap D_{\beta}\right)^{\circ} \subset \bar{U} \cap D_{\beta}^{\circ} \subset \bar{U} \cap E_{\alpha, 1},
$$


a contradiction. And if $i_{\beta}=1$ then we even have $U \cap D_{\beta}=\emptyset$.

Consequently, as $\kappa$ is regular, we have $\left|\bigcup\left\{D \cap D_{\beta}: \beta<\alpha\right\}\right|<\kappa$, hence $D_{\alpha}=U \cap D \backslash \bigcup\left\{D_{\beta}: \beta<\alpha\right\} \in \mathcal{D}$ and $y_{\alpha} \in D_{\alpha}^{\circ}$. Let us now put $i_{\alpha}=1$. Then $y_{\alpha} \in D_{\alpha}{ }^{\circ} \subset \overline{D_{\alpha}} \subset \overline{E_{\alpha+1,1}}$ implies $y_{\alpha} \in \overline{E_{\alpha+1,0}} \cap \overline{E_{\alpha+1,1}}$, hence (2.3) remains valid for $\alpha+1$.

To show the same for (2.2), note first that $D_{\beta}{ }^{\circ} \subset \overline{E_{\alpha, 1}}$, hence $\overline{D_{\alpha}} \cap$ $D_{\beta}{ }^{\circ}=\emptyset$ holds for each $\beta<\alpha$. Moreover, $D_{\alpha}{ }^{\circ} \subset \bar{U}$ implies $D_{\alpha}{ }^{\circ} \cap D_{\beta}=$ $\emptyset$ for any $\beta<\alpha$ with $i_{\beta}=1$, which together with $D_{\alpha} \cap D_{\alpha}{ }^{\circ}=\emptyset$ yields

$$
\left(E_{\alpha, 0} \cup D_{\alpha}^{\circ}\right) \cap\left(E_{\alpha, 1} \cup D_{\alpha}\right)=E_{\alpha+1,0} \cap E_{\alpha+1,1}=\emptyset .
$$

Of course, if $j=1$ then we shall have $i_{\alpha}=0$.

After having completed the inductive construction, it is trivial to conclude that $E_{\kappa, 0}$ and $E_{\kappa, 1}$ are two disjoint dense subsets of $X$.

We shall use lemma 2.4 in the proof of our main result, in the induction step of a procedure where we move from $n$-resolvability to $n+1$-resolvability.

In our following preliminary result, rather than the extent $\mathrm{e}(X)$, its "hat" version $\widehat{\mathrm{e}}(X)$ will appear. We recall that $\widehat{\mathrm{e}}(X)$ is defined as the smallest cardinal $\lambda$ such that $X$ has no closed-and-discrete subset of size $\lambda$. Thus we clearly have $\widehat{\mathrm{e}}(X) \leq \mathrm{e}(X)^{+}$, moreover $\widehat{\mathrm{e}}(X) \leq \kappa$ is simply equivalent with the statement that for every set $A \in[X]^{\kappa}$ we have $A^{\prime} \neq \emptyset$. We start with defining an auxiliary concept that will be needed in this result.

Definition 2.5. Let $X$ be a topological space and $\kappa$ be a cardinal. A subset $H \subset X$ is called $\kappa$-approachable in $X$ iff there are $\kappa$ many pairwise disjoint sets $\left\{X_{\alpha}: \alpha<\kappa\right\} \subset[X]^{\kappa}$ such that

$$
\forall Y \in\left[X_{\alpha}\right]^{\kappa}\left(Y^{\circ} \neq \emptyset\right) \text { and } H=\left(X_{\alpha}\right)^{\circ}
$$

hold true for all $\alpha<\kappa$.

The following lemma shows that this definition is not empty.

Lemma 2.6. Assume that $\kappa$ is a regular cardinal and $X$ is a space for which $\widehat{\mathrm{e}}(X) \leq \kappa$. Then

(1) $A \in[X]^{\kappa}$ and $\left|A^{\prime}\right|<\kappa$ imply $A^{\circ} \neq \emptyset$;

(2) if $A \in[X]^{\kappa}$ is such that $\left|A^{\circ}\right|<\kappa$ and $B^{\circ} \neq \emptyset$ for all $B \in[A]^{\kappa}$ then there is a subset $H \subset A^{\circ}$ that is $\kappa$-approachable in $X$.

Proof. (1) Every point $x \in A^{\prime} \backslash A^{\circ}$ has an open neighbourhood $U_{x}$ such that $\left|U_{x} \cap A\right|<\kappa$. Then $U=\bigcup\left\{U_{x}: x \in A^{\prime} \backslash A^{\circ}\right\}$ covers $A^{\prime} \backslash A^{\circ}$ and $|A \cap U|<\kappa$ because $\kappa$ is regular. So we have $|A \backslash U|=\kappa$ and hence $\emptyset \neq(A \backslash U)^{\prime}=A^{\circ}$ by $\kappa \geq \widehat{\mathrm{e}}(X)$. 
(2) We start by fixing $\kappa$ pairwise disjoint sets $\left\{A_{\alpha}: \alpha<\kappa\right\} \subset[A]^{\kappa}$ and for any $\alpha<\beta<\kappa$ we write

$$
A_{\alpha, \beta}=\bigcup_{\alpha \leq \nu<\beta} A_{\nu}
$$

For fixed $\alpha<\kappa$ the sequence

$$
\left\{A_{\alpha, \beta}{ }^{\circ}: \beta \in \kappa \backslash \alpha\right\}
$$

is increasing and hence must stabilize since $\left|A^{\circ}\right|<\kappa$. This means that there is an ordinal $f(\alpha)<\kappa$ such that

$$
A_{\alpha, \beta}^{\circ}=A_{\alpha, f(\alpha)}^{\circ}
$$

for all $\beta \in \kappa \backslash f(\alpha)$. Similarly, the sequence

$$
\left\{A_{\alpha, f(\alpha)^{\circ}}: \alpha<\kappa\right\}
$$

is decreasing and hence it stabilizes: There is an ordinal $\alpha^{*}<\kappa$ such that

$$
A_{\alpha^{*}, f\left(\alpha^{*}\right)}^{\circ}=A_{\alpha, f(\alpha)^{\circ}}
$$

whenever $\alpha^{*} \leq \alpha<\kappa$. We claim that the set $H=A_{\alpha^{*}, f\left(\alpha^{*}\right)}{ }^{\circ}$ is $\kappa$ approachable in $X$.

To see this, choose $I \in\left[\kappa \backslash \alpha^{*}\right]^{\kappa}$ in such a way that for any $\alpha, \beta \in I$ with $\alpha<\beta$ we have $f(\alpha)<\beta$. This is possible because $\kappa$ is regular. Then the sets

$$
\left\{A_{\alpha, f(\alpha)}: \alpha \in I\right\} \subset[X]^{\kappa}
$$

are pairwise disjoint and, by definition, for all $\alpha \in I$ we have both

$$
\forall B \in\left[A_{\alpha, f(\alpha)}\right]^{\kappa}\left(B^{\circ} \neq \emptyset\right) \text { and } A_{\alpha, f(\alpha)}^{\circ}=H .
$$

Our next resolvability result uses $\kappa$-approachable sets in an essential way.

Theorem 2.7. Assume that $X$ is a space, $\kappa=|X|$ is a regular cardinal, moreover $\mathcal{H}$ is a disjoint family of sets $\kappa$-approachable in $X$ such that $\cup \mathcal{H}$ is dense in $X$. Then the space $X$ is $\kappa$-resolvable.

Proof. For each $H \in \mathcal{H}$ let us fix a disjoint family $\left\{A_{H, \alpha}: \alpha<\kappa\right\} \subset$ $[X]^{\kappa}$ which witnesses that $H$ is $\kappa$-approachable in $X$, i.e.

$$
\forall B \in\left[A_{H, \alpha}\right]^{\kappa}\left(B^{\circ} \neq \emptyset\right) \text { and } A_{H, \alpha}{ }^{\circ}=H
$$

for all $\alpha<\kappa$.

Note that if $H, K \in \mathcal{H}$ and $\alpha, \beta \in \kappa$ with $\langle H, \alpha\rangle \neq\langle K, \beta\rangle$ then we have $\left|A_{H, \alpha} \cap A_{K, \beta}\right|<\kappa$. Indeed, if $H=K$ then $A_{H, \alpha} \cap A_{K, \beta}=\emptyset$. And if 
$H \neq K$ then $\left|A_{H, \alpha} \cap A_{K, \beta}\right|=\kappa$ would imply $\emptyset \neq\left(A_{H, \alpha} \cap A_{K, \beta}\right)^{\circ} \subset H \cap K$, contradicting $H \cap K=\emptyset$. This means that the family

$$
\mathcal{A}=\left\{A_{H, \alpha}: H \in \mathcal{H}, \alpha<\kappa\right\} \subset[X]^{\kappa}
$$

is almost disjoint.

But $|\mathcal{H}| \leq|X|=\kappa$ implies $|\mathcal{A}|=\kappa$, and then by the regularity of $\kappa$ it follows that $\mathcal{A}$ is also essentially disjoint. In other words, this means that for every pair $\langle H, \alpha\rangle$ there is a set $F_{H, \alpha} \in\left[A_{H, \alpha}\right]^{<\kappa}$ such that the collection

$$
\left\{B_{H, \alpha}=A_{H, \alpha} \backslash F_{H, \alpha}:\langle H, \alpha\rangle \in \mathcal{H} \times \kappa\right\}
$$

is already disjoint. Note also that for each $\langle H, \alpha\rangle \in \mathcal{H} \times \kappa$ we have

$$
B_{H, \alpha}{ }^{\circ}=A_{H, \alpha}{ }^{\circ}=H \text {. }
$$

We claim that for every $\alpha<\kappa$ the set

$$
D_{\alpha}=\bigcup\left\{B_{H, \alpha}: H \in \mathcal{H}\right\}
$$

is dense in $X$. Indeed, for any $U \in \tau^{+}(X)$ there is a set $H \in \mathcal{H}$ with $H \cap U \neq \emptyset$, so we may pick a point $x \in H \cap U$. But then $x \in H=B_{H, \alpha}^{\circ}$ implies $\left|U \cap B_{H, \alpha}\right|=\kappa$, consequently $U \cap D_{\alpha} \neq \emptyset$. Thus $\left\{D_{\alpha}: \alpha<\kappa\right\}$ is a family of $\kappa$ many pairwise disjoint dense sets in $X$.

$>$ From lemma 2.6 and theorem 2.7 we may immediately deduce the following corollary that will be needed in the proof of theorem 3.1. Maybe ironically, this does not even mention $\kappa$-approachable sets, but its proof does.

Corollary 2.8. Assume that $\kappa$ is a regular cardinal and $X$ is a space for which

$$
\widehat{\mathrm{e}}(X) \leq|X|=\kappa
$$

If the family

$$
\mathcal{A}^{\prime}=\left\{A^{\prime}: A \in[X]^{\kappa} \text { and }\left|A^{\prime}\right|<\kappa\right\}
$$

is a $\pi$-network in $X$ then the space $X$ is $\kappa$-resolvable.

Proof. By lemma 2.6 every member $A^{\prime}$ of $\mathcal{A}^{\prime}$ includes a set that is $\kappa$ approachable in $X$, hence if $\mathcal{A}^{\prime}$ is a $\pi$-network in $X$ then so is the family $\mathcal{G}$ of all the sets that are $\kappa$-approachable in $X$. But then the union of any maximal disjoint subfamily $\mathcal{H} \subset \mathcal{G}$ is clearly dense in $X$, hence all the assumptions of theorem 2.7 are satisfied.

We now turn to another circle of preliminary results that will be used in the proof of our main theorem 3.1. Again, we have to start with some definitions. 
Definition 2.9. If $X$ is a topological space, $Y \subset X$, and $\kappa$ is an infinite cardinal, then we call

$$
\bar{Y}^{<\kappa}=\bigcup\left\{\bar{S}: S \in[Y]^{<\kappa}\right\}
$$

the $<\kappa$-closure of $Y$ in $X$. We say that $Y$ is $<\kappa$-closed in $X$ iff $Y=\bar{Y}^{<\kappa}$. If $\kappa=\mu^{+}$then instead of $<\mu^{+}$-closure (resp. $<\mu^{+}$-closed) we simply say $\mu$-closure (resp. $\mu$-closed).

Definition 2.10. A chain decomposition of length $\beta$ (for some ordinal $\beta$ ) of a set $X$ is an increasing and continuous sequence $s=$ $\left\langle X_{\alpha}: \alpha<\beta\right\rangle$ such that $X=\bigcup\left\{X_{\alpha}: \alpha<\beta\right\}$. (Continuity means that $X_{\delta}=\bigcup\left\{X_{\alpha}: \alpha<\delta\right\}$ holds for any limit ordinal $\delta<\beta$. Since we also consider 0 a limit ordinal, this implies $X_{0}=\emptyset$.)

Clearly, if $s=\left\langle X_{\alpha}: \alpha<\beta\right\rangle$ is a chain decomposition of $X$ and $Y \subset X$ then $s \uparrow Y=\left\langle Y \cap X_{\alpha}: \alpha<\beta\right\rangle$ is a chain decomposition of $Y$. Moreover, if $C \subset \beta$ is a cub (closed and unbounded) subset of $\beta$ and $C=\left\{\gamma_{i}: i<\delta\right\}$ is the increasing enumeration of $C$ then $s[C]=\left\langle X_{\gamma_{i}}: i<\delta\right\rangle$ is again a chain decomposition of $X$.

Lemma 2.11. Assume that $\kappa=\operatorname{cf}(\kappa) \leq \lambda$ are infinite cardinals and $X$ is a topological space with $|X|=\lambda$. Then $X$ has a chain decomposition $s=\left\langle X_{\alpha}: \alpha<\operatorname{cf}(\lambda)\right\rangle$ such that $\left\{X_{\alpha}: \alpha<\operatorname{cf}(\lambda)\right\} \subset[X]^{<\lambda}$, moreover (C1) $X_{\alpha} \cap{\overline{X \backslash X_{\alpha}}}^{<\kappa} \subset{\overline{X_{\alpha+1} \backslash X_{\alpha}}}^{<\kappa}$ for each $\alpha<\operatorname{cf}(\lambda)$.

If, in addition, $X$ is $\pi$-regular and not $\mathrm{cf}(\lambda)$-resolvable then there are a cub set $C \subset \operatorname{cf}(\lambda)$ and a regular closed set $Y \in \mathrm{RC}^{+}(X)$ such that we also have

(C2) $Y \backslash X_{\alpha}$ is $<\kappa$-closed for each $\alpha \in C$.

Hence $Y$ has a chain-decomposition $\left\{Y_{\alpha}: \alpha<\operatorname{cf}(\lambda)\right\} \subset[Y]^{<\lambda}$ such that

$$
Y \backslash Y_{\alpha} \text { is }<\kappa \text {-closed for all } \alpha<\kappa
$$

Proof. Let us consider first every pair $\langle x, S\rangle$ such that $x \notin S$ but $x \in$ $\bar{S}^{<\kappa}$ and assign to this pair $\langle x, S\rangle$ a set $A(x, S) \in[S]^{<\kappa}$ with $x \in$ $\overline{A(x, S)}$. Moreover, choose a chain decomposition $\left\{Z_{\alpha}: \alpha<\operatorname{cf}(\lambda)\right\} \subset$ $[X]^{<\lambda}$ of $X$ in an arbitrary manner. Then we define the sequence $\left\langle X_{\alpha}: \alpha<\mathrm{cf}(\lambda)\right\rangle$ by transfinite recursion on $\alpha$ as follows:

(i) $X_{0}=\emptyset$;

(ii) $X_{\alpha}=\bigcup\left\{X_{\beta}: \beta<\alpha\right\}$ if $\alpha>0$ is limit;

(iii) $X_{\alpha+1}=X_{\alpha} \cup Z_{\alpha} \cup \bigcup\left\{A\left(x, X \backslash X_{\alpha}\right): x \in X_{\alpha} \cap{\overline{X \backslash X_{\alpha}}}^{<\kappa}\right\}$. 
Since $\kappa \leq \lambda$ is regular, we can show by an easy transfinite induction that $\left|X_{\alpha}\right|<\lambda$ for all $\alpha<\operatorname{cf}(\lambda)$, moreover condition (C1) obviously follows from case (iii) of our definition. This proves the first half of the lemma.

Now assume that, in addition, $X$ is not $\operatorname{cf}(\lambda)$-resolvable. For each $A \subset \operatorname{cf}(\lambda)$ let us set

$$
R_{A}=\bigcup_{\alpha \in A}\left(X_{\alpha+1} \backslash X_{\alpha}\right)
$$

If $R_{A}$ would be dense in $X$ for all $A \in[\operatorname{cf}(\lambda)]^{\operatorname{cf}(\lambda)}$ then clearly $X$ would be $\operatorname{cf}(\lambda)$-resolvable, hence there is a cofinal $A \subset \operatorname{cf}(\lambda)$ and an open set $U \in \tau^{+}(X)$ with $U \cap R_{A}=\emptyset$.

We claim that then for every closed set $F \subset U$ and for every $\alpha \in A$ we have that $F \backslash X_{\alpha}$ is $<\kappa$-closed. Indeed, assume on the contrary that $x \in{\overline{F \backslash X_{\alpha}}}^{<\kappa} \cap X_{\alpha}$. Then, by $(\mathrm{C} 1)$, there is a set $S \in\left[X_{\alpha+1} \backslash X_{\alpha}\right]^{<\kappa}$ with $x \in \bar{S}$. Since $x \in F \subset U$, this implies $U \cap S \neq \emptyset$, which contradicts $U \cap R_{A}=\emptyset$, as $\alpha \in A$ and $S \subset X_{\alpha+1} \backslash X_{\alpha}$.

Now, if $X$ is also $\pi$-regular then there is a regular closed set $Y \in$ $\mathrm{RC}^{+}(X)$ with $Y \subset U$. Let us consider the set

$$
C=\left\{\alpha<\operatorname{cf}(\lambda): Y \backslash X_{\alpha} \text { is }<\kappa \text {-closed }\right\} .
$$

$C$ is clearly closed in $\operatorname{cf}(\lambda)$ and $A \subset C$ by the above, hence $C$ is cub in $\operatorname{cf}(\lambda)$. This completes the proof of lemma 2.11.

We have one more preparatory result involving chain decompositions that will be used in the proof of our main theorem.

Lemma 2.12. Assume that $Y$ is a $\pi$-regular space that is not $\omega$ resolvable. Then for every chain decomposition $\left\{Y_{\alpha}: \alpha<\mu\right\}$ of $Y$ there are $T \in \mathrm{RC}^{+}(Y)$ and a dense subset $Z \subset T$ such that

$$
\overline{Y_{\alpha} \cap Z} \subset Y_{\alpha} \text { for all } \alpha<\mu \text {. }
$$

Proof. By the continuity of chain decompositions, for each point $x \in Y$ there is a unique ordinal $\alpha(x)<\mu$ such that

$$
x \in Y_{\alpha(x)+1} \backslash Y_{\alpha(x)} .
$$

For any subset $A \subset Y$ let us define

$$
A^{*}=\left\{x \in A: x \notin \overline{A \cap Y_{\alpha(x)}}\right\} .
$$

We claim that $A^{*}$ is dense in $A$ for every $A \subset Y$.

Indeed, if $U$ is open and $U \cap A \neq \emptyset$, then pick $a \in U \cap A$ such that $\alpha(a)$ is minimal. Then $a \in A^{*}$ because by the minimality of $\alpha(a)$ we have $U \cap Y_{\alpha(a)}=\emptyset$. 
Let us now define the sets $\left\{D_{j}: j<\omega\right\}$ by means of the following recursive formula:

$$
D_{j}=\left(Y \backslash \bigcup_{i<j} D_{i}\right)^{*}
$$

The pairwise disjoint sets $\left\{D_{j}: j<\omega\right\}$ cannot be all dense in $Y$ because $Y$ is not $\omega$-resolvable, but $D_{0}=Y^{*}$ is dense. So there is $m \in \omega$ such that $D_{m}$ is dense but $D_{m+1}$ is not, hence $U \cap D_{m+1}=\emptyset$ for some $U \in \tau^{+}(X)$. Now, pick $T \in \mathrm{RC}^{+}(Y)$ with $T \subset U$.

Then $U \cap D_{m+1}=U \cap\left(Y \backslash \bigcup_{i \leq m} D_{i}\right)^{*}=\emptyset$ implies

$$
T \subset U \subset \bigcup_{j \leq m} D_{j}
$$

and clearly $Z=T \cap D_{m}$ is dense in $T$.

Now it remains to show that

$$
\overline{Z \cap Y_{\alpha}} \subset Y_{\alpha} \text { for all } \alpha<\mu \text {. }
$$

To see this, fix $\alpha<\mu$ and consider first any point $x \in T$. Then $x \in D_{j}$ for some $j \leq m$ by (2.6). This means that $x \in\left(Y \backslash \bigcup_{i<j} D_{i}\right)^{*}$, i.e. $x \notin \overline{\left(Y \backslash \bigcup_{i<j} D_{i}\right) \cap Y_{\alpha(x)}}$. But $D_{m} \subset Y \backslash \bigcup_{i<j} D_{i}$, hence we have $x \notin \overline{D_{m} \cap Y_{\alpha(x)}}$.

On the other hand, for every point $x \in \overline{Z \cap Y_{\alpha}}(\subset T)$ we have $x \in$ $\overline{D_{m} \cap Y_{\alpha}}$ because $Z \subset D_{m}$. This together with $x \notin \overline{D_{m} \cap Y_{\alpha(x)}}$ implies $\alpha(x)<\alpha$ because the sets $Y_{\beta}$ are increasing. So,by the definition of $\alpha(x)$ we have $x \in Y_{\alpha(x)+1} \subset Y_{\alpha}$, and this means that $\overline{Z \cap Y_{\alpha}} \subset Y_{\alpha}$.

Our next preliminary results will be used in the proof of theorem 4.1, a stepping-up result concerning resolvability of certain spaces.

Lemma 2.13. Assume that $\kappa$ is an infinite cardinal, $X$ a topological space, and we have a disjoint subfamily $\mathcal{H} \subset \mathfrak{R}_{\kappa}(X)$ such that for each $U \in \tau^{+}(X)$

$$
|\{H \in \mathcal{H}: H \cap U \neq \emptyset\}|=\kappa^{+} .
$$

Then $X$ is $\kappa^{+}$-resolvable.

Proof of lemma 2.13. Obviously, $|\mathcal{H}|=\kappa^{+}$, so we can fix a one-one enumeration $\mathcal{H}=\left\{H_{\xi}: \xi<\kappa^{+}\right\}$of $\mathcal{H}$. Every $H_{\xi}$ is $\kappa$-resolvable, and so has a partition

$$
H_{\xi}=\bigcup^{*}\left\{H_{\xi}^{i}: i<\xi\right\}
$$

into dense subsets. Then for every $i<\kappa^{+}$the set

$$
D^{i}=\bigcup\left\{H_{\xi}^{i}: i<\xi<\kappa^{+}\right\} .
$$


is dense in $X$. Indeed, for each $U \in \tau^{+}(X)$ by our assumption there is $\xi>i$ with $U \cap H_{\xi} \neq \emptyset$. But $H_{\xi}^{i}$ is dense in $H_{\xi}$, so we have $U \cap D^{i} \supset$ $U \cap H_{\xi}^{i} \neq \emptyset$ as well. As the dense sets $\left\{D^{i}: i<\kappa^{+}\right\}$are pairwise disjoint, our proof is complete.

To formulate the following corollary of lemma 2.13, we need one more definition.

Definition 2.14. Let $X$ be any topological space and $\kappa$ an infinite cardinal. A (necessarily closed) subset $F \subset X$ is called $\kappa$-nice in $X$ if there is a disjoint family $\left\{A_{\alpha}: \alpha<\kappa^{+}\right\} \subset \mathfrak{R}_{\kappa}(X)$ such that

$$
F=\bigcap_{\alpha<\kappa^{+}} \overline{\bigcup\left\{A_{\beta}: \beta \in \kappa^{+} \backslash \alpha\right\}} \text {. }
$$

Following the terminology of [5], we call a space $\lambda$-compact if every subset of it of cardinality $\lambda$ has a complete accumulation point.

Corollary 2.15. Let $\kappa$ be an infinite cardinal and $X$ be a $\kappa^{+}$-compact space. If there is a disjoint family $\mathcal{F}$ of both $\kappa$-resolvable and $\kappa$-nice subsets of $X$ such that $|\mathcal{F}| \leq \kappa^{+}$and $\bigcup \mathcal{F}$ is dense in $X$, then $X$ has $a \kappa^{+}$-resolvable open subset.

Proof. If for every $U \in \tau^{+}(X)$ we have $|\{F \in \mathcal{F}: F \cap U \neq \emptyset\}|=\kappa^{+}$ then $X$ itself is $\kappa^{+}$-resolvable by lemma 2.13. So assume that $U \in$ $\tau^{+}(X)$ is such that $\mathfrak{F}^{*}=\{F \in \mathcal{F}: U \cap F \neq \emptyset\}$ has cardinality $\leq \kappa$.

For each $F \in \mathcal{F}^{*}$ let us fix a disjoint family

$$
\left\{A_{\alpha}^{F}: \alpha<\kappa^{+}\right\} \subset \mathfrak{R}_{\kappa}(X)
$$

witnessing that $F$ is nice, as required in definition 2.14. We claim that for every pair $\{F, G\} \in\left[\mathcal{F}^{*}\right]^{2}$ there is an $\alpha=\alpha(F, G)<\kappa^{+}$such that

$$
\bigcup\left\{A_{\beta}^{F}: \beta \in \kappa^{+} \backslash \alpha\right\} \cap \bigcup\left\{A_{\beta}^{G}: \beta \in \kappa^{+} \backslash \alpha\right\}=\emptyset .
$$

Indeed, otherwise we could select a set $I \in\left[\kappa^{+}\right]^{\kappa^{+}}$and distinct points $\left\{x_{\alpha}: \alpha \in I\right\}$ such that

$$
x_{\alpha} \in \bigcup\left\{A_{\beta}^{F}: \beta \in \kappa^{+} \backslash \alpha\right\} \cap \bigcup\left\{A_{\beta}^{G}: \beta \in \kappa^{+} \backslash \alpha\right\}
$$

whenever $\alpha \in I$. But then $\left\{x_{\alpha}: \alpha \in I\right\}^{\circ} \neq \emptyset$ would be a subset of

$$
\bigcap_{\alpha \in I} \overline{\bigcup\left\{A_{\beta}^{F}: \beta \in \kappa^{+} \backslash \alpha\right\}} \cap \bigcap_{\alpha \in I} \overline{\bigcup\left\{A_{\beta}^{G}: \beta \in \kappa^{+} \backslash \alpha\right\}}=F \cap G,
$$

contradicting $F \cap G=\emptyset$. 
Now, $\left|\mathcal{F}^{*}\right| \leq \kappa$ implies that there is an ordinal $\gamma<\kappa^{+}$such that $\alpha(F, G)<\gamma$ for all pairs $\{F, G\} \in\left[\mathcal{F}^{*}\right]^{2}$. Consequently, the elements of the family

$$
\mathcal{J}=\left\{A_{\alpha}^{F}: F \in \mathcal{F}^{*} \text { and } \alpha \in \kappa^{+} \backslash \gamma\right\} \subset \mathfrak{R}_{\kappa}(X)
$$

are pairwise disjoint and, by our assumptions, both $\bigcup \mathcal{F}^{*} \cap U$ and $\bigcup \mathcal{J} \cap U$ are dense in $U$.

Thus, for every $V \in \tau^{+}(U)$ there is $F \in \mathcal{F}^{*}$ for which $V \cap F \neq \emptyset$. But this clearly implies that $\left|\left\{\alpha \in \kappa^{+} \backslash \gamma: V \cap A_{\alpha}^{F} \neq \emptyset\right\}\right|=\kappa^{+}$, hence $U$ and the family

$$
\mathcal{H}=\mathcal{J} \uparrow U=\left\{U \cap A_{\alpha}^{F}: F \in \mathcal{F}^{*} \text { and } \alpha \in \kappa^{+} \backslash \gamma\right\}
$$

satisfy the assumptions of lemma 2.13 , consequently $U$ is $\kappa^{+}$-resolvable. 


\section{The Main Result}

We are now ready to formulate and prove our main result.

Theorem 3.1. Let $\kappa$ be an uncountable regular cardinal. Then every regular space $X$ satisfying

$$
\Delta(X) \geq \kappa \geq \widehat{\mathrm{e}}(X)
$$

is $\omega$-resolvable. Consequently, every regular space $X$ that satisfies $\Delta(X)>$ $\mathrm{e}(X)$ is $\omega$-resolvable.

Proof. For convenience, after fixing $\kappa$, we denote by $\mathcal{C}$ the class of all regular spaces $X$ that satisfy $\Delta(X) \geq \kappa \geq \widehat{\mathrm{e}}(X)$. Clearly, the class $\mathcal{C}$ is regular closed hereditary, that is for every $X \in \mathcal{C}$ we have $\mathrm{RC}^{+}(X) \subset \mathcal{C}$. By corollary 2.2 , to prove that all members of $\mathcal{C}$ are $\omega$-resolvable it suffices to show that every $X \in \mathcal{C}$ with $|X|=\Delta(X)$ is $\omega$-resolvable.

To achieve this, for any cardinal $\lambda \geq \kappa$ we set

$$
\mathcal{C}_{\lambda}=\{X \in \mathcal{C}:|X|=\Delta(X)=\lambda\},
$$

and then we prove by induction on $\lambda \geq \kappa$ that

$\left(*_{\lambda}\right)$ every member of $\mathcal{C}_{\lambda}$ is $\omega$-resolvable.

So let us assume now that $\lambda \geq \kappa$ and $\left(*_{\mu}\right)$ holds whenever $\kappa \leq$ $\mu<\lambda$. Clearly, this implies that every space $X \in \mathcal{C}$ with $|X|<\lambda$ is $\omega$-resolvable.

To deduce $\left(*_{\lambda}\right)$ from this, by theorem 2.3 , it suffices to show that every member of $\mathcal{C}_{\lambda}$ is $n$-resolvable for all $n \in \omega \backslash\{0\}$. This, in turn, will be proved by a subinduction on $n \in \omega \backslash\{0\}$. Therefore we assume from here on that for some $n>0$ we have

$\left(o_{n}\right)$ every member of $\mathcal{C}_{\lambda}$ is $n$-resolvable

and we want to deduce $\left(o_{n+1}\right)$ from this. (Of course, $\left(\circ_{1}\right)$ holds trivially.)

To prove $\left(\circ_{n+1}\right)$, we observe first that the class $\mathcal{C}_{\lambda}$ is also regular closed hereditary, hence by corollary 2.2 again, $\left(o_{n+1}\right)$ is implied by the following seemingly weaker statement:

$\left(o_{n+1}^{\prime}\right)$ every member of $\mathcal{C}_{\lambda}$ has an $(n+1)$-resolvable subspace.

Now, the proof of $\left(\mathrm{o}_{n+1}^{\prime}\right)$ branches into two: Namely, the initial case $\lambda=\kappa$ and the case $\lambda>\kappa$ of the induction on $\lambda$ are handled differently.

Case 1. $\lambda=\kappa$

Consider any $X \in \mathcal{C}_{\kappa}$ and recall that our aim is to show that $X$ has an $(n+1)$-resolvable subspace. If $X$ is $\kappa$-resolvable then we are done. 
Otherwise by lemma 2.6 there is $Q \in \mathrm{RC}^{+}(X)$ such that

$$
\left|A^{\prime}\right|=\kappa \text { for all } A \in[Q]^{\kappa} \text {. }
$$

It easily follows from (3.1) that for every $Y \in[Q]^{<\kappa}$ and for every $B \in \mathrm{RC}^{+}(Q)\left(\subset \mathrm{RC}^{+}(X)\right)$ we have $B \backslash Y \in \mathcal{C}_{\kappa}$. Consequently, if $Y \cap B$ is dense in $B$ then $B$ is $(n+1)$-resolvable because $B \backslash Y$ is $n$-resolvable by $\left(o_{n}\right)$. So from here on we can assume that

$$
\text { every set in }[Q]^{<\kappa} \text { is nowhere dense. }
$$

Let us now apply lemma 2.11 to the space $Q$ and with the choice $\kappa=\lambda$. This yields us some $Y \in \mathrm{RC}^{+}(Q)$ and a chain decomposition $\left\{Y_{\alpha}: \alpha<\kappa\right\} \subset[Y]^{<\kappa}$ of $Y$ such that

$$
Y \backslash Y_{\alpha} \text { is }<\kappa \text {-closed for each } \alpha<\kappa \text {. }
$$

If $Y$ happens to be $\omega$-resolvable (or just $(n+1)$-resolvable) then, of course, we are done. Otherwise we may apply lemma 2.12 to the chain decomposition $\left\{Y_{\alpha}: \alpha<\kappa\right\}$ of $Y$ to obtain $T \in \mathrm{RC}^{+}(Y)$ with a dense subset $Z \subset T$ such that

$$
\overline{Y_{\alpha} \cap Z} \subset Y_{\alpha} \text { for all } \alpha<\kappa .
$$

Write $R_{\alpha}=Y_{\alpha+1} \backslash Y_{\alpha}$ for $\alpha<\kappa$. For each $x \in Y$ we let $\alpha(x) \in \kappa$ be the unique ordinal with $x \in R_{\alpha(x)}$. We call a subset $E \subset Y$ rare iff $\left|E \cap R_{\alpha}\right| \leq 1$ for all $\alpha<\kappa$. It is immediate from (3.3) and (3.4) that every rare subset $E$ of $Z$ of size $<\kappa$ is closed-and-discrete, i.e. satisfies $E^{\prime}=\emptyset$.

Let us now consider the family

$$
\mathcal{D}=\left\{D \in[Z]^{\kappa}: D \text { is discrete and } \forall E \in[D]^{<\kappa}\left(E^{\prime}=\emptyset\right)\right\} .
$$

The derived set $E^{\prime}$ of $E$ above is always meant to be taken in $T$ (or equivalently in $X$ ), not in $Z$. It is obvious from the definition that for every $D \in \mathcal{D}$ we have $D^{\prime}=D^{\circ}$ and $[D]^{\kappa} \subset \mathcal{D}$.

Claim 3.1.1. For every $D \in \mathcal{D}$ we have $\Delta\left(D^{\circ}\right)=\kappa$, consequently $D^{\circ} \in \mathcal{C}_{\kappa}$.

Proof of the claim. Assume that $G$ is any open set with $G \cap D^{\circ} \neq \emptyset$ and pick a point $x \in G \cap D^{\circ}$. By the regularity of the space $X$ there is an open set $H$ such that $x \in H \subset \bar{H} \subset G$. Then we have $|H \cap D|=\kappa$, as $x \in D^{\circ}$, and hence $\left|(H \cap D)^{\circ}\right|=\kappa$ by (3.1). But we clearly have

$$
(H \cap D)^{\circ} \subset \bar{H} \cap D^{\circ} \subset G \cap D^{\circ},
$$

hence $\left|G \cap D^{\circ}\right|=\kappa$ and therefore $\Delta\left(D^{\circ}\right)=\kappa$. Since $D^{\circ}$ is closed in $X$ it is obvious that $\widehat{\mathrm{e}}\left(D^{\circ}\right) \leq \widehat{\mathrm{e}}(X) \leq \kappa$ and hence $D^{\circ} \in \mathcal{C}_{\kappa}$. 
We note that this proof used the full force of the regularity of our space.

Claim 3.1.2. Assume that $V \in \mathrm{RC}^{+}(T)$ and the set $S \subset V \cap Z$ is dense in $V$. If $S$ is not $\kappa$-resolvable then there is some $D \in \mathcal{D}$ such that $D \subset S$.

Proof of the claim. Since every member of $\tau^{+}(S)$ is somewhere dense in $V$ and hence in $Q$, it follows from (3.2) that $\Delta(S)=\kappa=\operatorname{cf}(\kappa)>\omega$. If $S$ is not $\kappa$-resolvable then [4, Theorem 2.2] implies that $S$ must have a (relatively) discrete subset $J$ of size $\kappa$. Clearly, there is $D \in[J]^{\kappa}$ that is rare. But then $D \in \mathcal{D}$ because, as was pointed out above, we have $E^{\prime}=\emptyset$ for all rare sets $E$ of size $<\kappa$.

By the (sub)inductive assumption $\left(o_{n}\right)$ we have a partition

$$
T=\bigcup_{i<n} Z_{i}
$$

of $T$ into $n$ pairwise disjoint dense subsets $Z_{i}$. Since $Z \subset T$ is also dense, it is not possible that $Z \cap Z_{i}$ is nowhere dense for all $i<n$. Thus we can assume, without loss of generality, that $Z \cap Z_{0}$ is somewhere dense, say it is dense in $V \in \mathrm{RC}^{+}(T)$.

If there is some $W \in \mathrm{RC}^{+}(V)$ for which $W \cap Z \cap Z_{0}$ is $\kappa$-resolvable (or just $(n+1)$-resolvable) then again we are done. Otherwise, by claim 3.1.2, for each $W \in \mathrm{RC}^{+}(V)$ the set $W \cap Z \cap Z_{0}$ includes a member of $\mathcal{D}$, hence we may assume that

$$
\mathcal{E}=\left\{D \in \mathcal{D}: D \subset V \cap Z \cap Z_{0}\right\} \text { is a } \pi \text {-network in } V .
$$

Now we distinguish two subcases.

\section{Subcase 1.}

$$
\mathcal{E}_{0}=\left\{D \in \mathcal{E}: D^{\circ} \cap Z \cap Z_{0}=\emptyset\right\}
$$

is a $\pi$-network in $V$.

In this case the family $\mathcal{F}=\left\{D^{\circ}: D \in \mathcal{E}_{0}\right\}$ is also a $\pi$-network in $V$ because $V$ is $\pi$-regular, hence by definition $\mathcal{F}$ is a $\pi$-network in $V \backslash\left(Z \cap Z_{0}\right)$ as well. But every $D^{\circ} \in \mathcal{F}$ is $n$-resolvable by claim 3.1.1 and $\left(o_{n}\right)$, hence so is $V \backslash\left(Z \cap Z_{0}\right)$ by theorem 2.1. This, however, implies that $V$ is $(n+1)$-resolvable because $V \cap Z \cap Z_{0}$ is also dense in $V$.

Subcase 2. $\mathcal{E}_{0}$ is not a $\pi$-network in $V$, i.e. there is $U \in \mathrm{RC}^{+}(V)$ such that if $D \in \mathcal{D}$ and $D \subset U \cap Z \cap Z_{0}$ then $D^{\circ} \cap U \cap Z \cap Z_{0} \neq \emptyset$. Now

$$
\mathcal{E}_{1}=\left\{D \in \mathcal{D}: D \subset U \cap Z \cap Z_{0}\right\}=\{D \in \mathcal{E}: D \subset U\}
$$


is a $\pi$-network in $U \cap Z \cap Z_{0}$. From this it follows that the set

$$
S=\left(U \cap Z \cap Z_{0}\right) \cap \bigcup\left\{D^{\circ}: D \in \mathcal{E}_{1}\right\}
$$

is dense in $U \cap Z \cap Z_{0}$.

Now, it is easy to check then that the space $U \cap Z \cap Z_{0}$, the cardinal $\kappa$, the family $\mathcal{E}_{1}$, and the dense subset $S$ of $\left(U \cap Z \cap Z_{0}\right)$ satisfy all the assumptions of lemma 2.4, hence $U \cap Z \cap Z_{0}$, and thus $U \cap Z_{0}$ as well, is 2-resolvable. But $U \backslash Z_{0}$ is clearly $n-1$-resolvable, and so it follows that $U$ is $(n+1)$-resolvable. This completes the proof of $\left(o_{n+1}^{\prime}\right)$ in the case $\lambda=\kappa$.

Case 2. $\lambda>\kappa$.

Recall that our aim is to show that every space $X \in \mathcal{C}_{\lambda}$ has an $(n+1)$ resolvable subspace. Assume first that there are $B \in \mathrm{RC}^{+}(X)$ and a dense subset $A$ of $B$ with $|A|<\lambda$ such that $B \backslash A$ is $\kappa$-closed. Then we have $\Delta(B \backslash A)=\lambda$ because $|A|<\lambda$ and $\widehat{\mathrm{e}}(B \backslash A) \leq \kappa$ because $B \backslash A$ is $\kappa$-closed, consequently $B \backslash A \in \mathcal{C}_{\lambda}$. So the (sub)inductive assumption $\left(\circ_{n}\right)$ implies that $B \backslash A$ is $n$-resolvable and hence $B$ is $(n+1)$-resolvable.

Thus we may assume from here on that

if $A \in[X]^{<\lambda}$ and $X \backslash A$ is $\kappa$-closed then $A$ is nowhere dense.

Let us now apply lemma 2.11 to the space $X$ and the cardinals $\lambda$ and $\kappa^{+}$. This is possible because $\lambda \geq \kappa^{+}$. This way we obtain $Y \in \mathrm{RC}^{+}(X)$ with a chain decomposition

$$
\left\{Y_{\alpha}: \alpha<\operatorname{cf}(\lambda)\right\} \subset[Y]^{<\lambda}
$$

of length $\operatorname{cf}(\lambda)$ such that for each $\alpha<\operatorname{cf}(\lambda)$ we have

$$
Y \backslash Y_{\alpha} \text { is } \kappa \text {-closed for each } \alpha<\lambda \text {. }
$$

Note that then each $Y_{\alpha}$ is nowhere dense by (3.6).

For any point $x \in Y$ we again define the ordinal $\alpha(x)<\operatorname{cf}(\lambda)$ by the formula $x \in Y_{\alpha(x)+1} \backslash Y_{\alpha(x)}$ and call a set $E \subset Y$ is rare iff $\mid E \cap\left(Y_{\alpha+1} \backslash\right.$ $\left.Y_{\alpha}\right) \mid \leq 1$ for all $\alpha<\operatorname{cf}(\lambda)$.

If $Y$ is $\omega$-resolvable then we are done. Otherwise we may apply lemma 2.12 to obtain $T \in \mathrm{RC}^{+}(Y)$ with a dense subset $D \subset T$ such that

$$
\overline{Y_{\alpha} \cap D} \subset Y_{\alpha} \text { for all } \alpha<\operatorname{cf}(\lambda) .
$$

We claim that $D$ has no rare subset of cardinality $\kappa$. This is because for any rare set $E \in[D]^{\kappa}$ we would have had $E^{\prime}=\emptyset$, contradicting $\widehat{\mathrm{e}}(X) \leq \kappa$. 
To see this, pick any point $x \in Y$. Then $x \notin \overline{D \cap Y_{\alpha(x)}} \subset Y_{\alpha(x)}$ by 3.8, moreover $x \notin \overline{E \backslash Y_{\alpha(x)+1}}$ because $Y \backslash Y_{\alpha(x)+1}$ is $\kappa$-closed by (3.7). But $\left|E \cap\left(Y_{\alpha(x)+1} \backslash Y_{\alpha(x)}\right)\right| \leq 1$, hence we clearly have $x \notin E^{\prime}$.

Consequently, if we got this far, i.e. no $(n+1)$-resolvable subspace has been found yet, then we must have $\operatorname{cf}(\lambda)<\kappa$. Indeed, since each $Y_{\alpha}$ is nowhere dense but $D$ is not, there are cofinally many $\alpha<\operatorname{cf}(\lambda)$ with $D \cap\left(Y_{\alpha+1} \backslash Y_{\alpha}\right) \neq \emptyset$. But then $\operatorname{cf}(\lambda) \geq \kappa$ would clearly imply the existence of a rare subset of $D$ of size $\kappa$.

Let us now put $T_{\alpha}=T \cap Y_{\alpha}$ and $Z_{\alpha}=\overline{\left(D \cap T_{\alpha+1}\right)} \backslash T_{\alpha}$ for $\alpha<\operatorname{cf}(\lambda)$. Then $Z_{\alpha} \subset T_{\alpha+1} \backslash T_{\alpha}$ by (3.8) and $Z_{\alpha}$ is $\kappa$-closed, being the intersection of a closed and a $\kappa$-closed set. This clearly implies $\widehat{\mathrm{e}}\left(Z_{\alpha}\right) \leq \kappa$.

Moreover, the set

$$
Z=\bigcup_{\alpha<\operatorname{cf}(\lambda)} Z_{\alpha} \subset T
$$

is dense in $T$ because $D \subset Z$. We also have $\widehat{\mathrm{e}}(Z) \leq \kappa$ because $\widehat{\mathrm{e}}\left(Z_{\alpha}\right) \leq \kappa$ for each $\alpha<\operatorname{cf}(\lambda)$ and $\operatorname{cf}(\lambda)<\kappa=\operatorname{cf}(\kappa)$.

The following observation will be crucial in the rest of our proof.

Claim 3.1.3. Every set $H \in[Z]^{\leq \kappa}$ is nowhere dense.

Proof of the claim. Let us fix $H \in[Z]^{\leq \kappa}$ and pick $\alpha<\operatorname{cf}(\lambda)$. Then we have $\overline{H \cap T_{\alpha}} \subset \overline{D \cap T_{\alpha}} \subset T_{\alpha}$ by (3.8) and $\overline{H \backslash T_{\alpha+1}} \subset T \backslash T_{\alpha+1}$ by (3.7). Moreover, we also have

$$
\overline{H \cap\left(T_{\alpha+1} \backslash T_{\alpha}\right)} \subset Z_{\alpha} \subset T_{\alpha+1} \backslash T_{\alpha}
$$

because $Z_{\alpha} \subset T_{\alpha+1} \backslash T_{\alpha}$ is $\kappa$-closed, hence

$$
\bar{H} \cap\left(T_{\alpha+1} \backslash T_{\alpha}\right)=\overline{H \cap\left(T_{\alpha+1} \backslash T_{\alpha}\right)} .
$$

This then implies that $\left\{\bar{H} \cap\left(T_{\alpha+1} \backslash T_{\alpha}\right): \alpha<\operatorname{cf} \lambda\right\}$ is a partition of $\bar{H}$ into relatively clopen subsets of size $<\lambda$. Consequently, for all $U \in \tau^{+}(\bar{H})$ we have $\Delta(U)<\lambda$, while for every $W \in \tau^{+}(X)$ we have $\Delta(W)=\lambda$. But this implies that $\operatorname{Int} \bar{H}=\emptyset$, i.e. $H$ (or, equivalently $\bar{H})$ is nowhere dense.

If there are an $\alpha<\operatorname{cf}(\lambda)$ and an $R \in \mathrm{RC}^{+}\left(Z_{\alpha}\right)$ with $\Delta(R) \geq \kappa(\geq$ $\widehat{\mathrm{e}}(R))$ then, as $R \in \mathcal{C}$ and $|R|<\lambda$, our inductive hypothesis implies that $R$ is $\omega$-resolvable, hence we are done.

Consequently, we may assume that

$$
\mathcal{P}_{\alpha}=\left\{U \in \tau^{+}\left(Z_{\alpha}\right):|U|<\kappa\right\}
$$


is a $\pi$-base of $Z_{\alpha}$ for each $\alpha<\operatorname{cf}(\lambda)$. For any $\alpha<\operatorname{cf}(\lambda)$ let $\mathcal{E}_{\alpha}$ be a maximal disjoint subfamily of $\mathcal{P}_{\alpha}$. It follows then that $E_{\alpha}=\bigcup \mathcal{E}_{\alpha}$ is a dense open subset of $Z_{\alpha}$, consequently

$$
E=\bigcup_{\alpha<\operatorname{cf}(\lambda)} E_{\alpha}
$$

is dense in $Z$ and hence in $T$.

Let us now put $F_{\alpha}=Z_{\alpha} \backslash E_{\alpha}$ for all $\alpha<\operatorname{cf}(\lambda)$ and

$$
F=\bigcup_{\alpha<\operatorname{cf}(\lambda)} F_{\alpha} .
$$

Since $F_{\alpha}$ is closed in $Z_{\alpha}$ we have $\widehat{\mathrm{e}}\left(F_{\alpha}\right) \leq \widehat{\mathrm{e}}\left(Z_{\alpha}\right) \leq \kappa$ and so, by $\operatorname{cf}(\lambda)<$ $\kappa$,

$$
\widehat{\mathrm{e}}(F) \leq \kappa
$$

as well.

We claim that $F$ is also dense in $Z$. Assume on the contrary that $F \cap V=\emptyset$ for some $V \in \mathrm{RC}^{+}(Z)$, i.e. $V \subset E$. Then $V \cap Z_{\alpha} \subset E_{\alpha}$ for each $\alpha<\operatorname{cf}(\lambda)$, hence $\mathcal{U}_{\alpha}=\left\{U \cap\left(V \cap Z_{\alpha}\right): U \in \mathcal{E}_{\alpha}\right\}$ yields a partition of $V \cap Z_{\alpha}$ into (relatively) clopen subsets of $V \cap Z_{\alpha}$. But $V \cap Z_{\alpha}$ is closed in $Z_{\alpha}$, consequently, $\widehat{\mathrm{e}}\left(Z_{\alpha}\right) \leq \kappa$ implies $\left|\mathcal{U}_{\alpha}\right|<\kappa$. But then we also have $\left|V \cap Z_{\alpha}\right|<\kappa$ because $|U|<\kappa$ for each $U \in \mathcal{U}_{\alpha}$ and $\kappa$ is a regular cardinal. This, in turn, implies $|V \cap Z|<\kappa$ because cf $\lambda<\kappa$. But $V \cap Z$ is somewhere dense and this contradicts claim 3.1.3. So $F$ is indeed dense in $Z$.

As $F$ is dense in $Z$, applying claim 3.1 .3 again we conclude that

$$
\lambda \geq \Delta(F)>\kappa \text {. }
$$

Putting (3.9) and (3.10) together, our inductive hypotheses, including $\left(o_{n}\right)$, imply that $F$ is $n$-resolvable, hence $Z$ is $(n+1)$-resolvable because $E \cap F=\emptyset$. Thus $\left(\circ_{n+1}^{\prime}\right)$ is verified and the proof is completed.

Let us now make a few comments on the assumptions of our main theorem 3.1. Although the uncountability of $\kappa$ was used in our proof when we referred to theorem 2.2 in [4, theorem 3.1 is valid for $\kappa=\omega$ as well. Indeed, to see this we note that $\widehat{\mathrm{e}}(X)=\omega$ just means that $X$ is countably compact, and Pytkeev proved in [8] that crowded countably compact regular spaces are even $\omega_{1}$-resolvable.

The question if the assumption on the regularity of $\kappa$ is essential is more interesting and we do not know the answer to it. We only have the following partial positive result in the case when $\kappa$ is a singular cardinal of countable cofinality. 
Theorem 3.2. Let $\kappa$ be a singular cardinal of countable cofinality. Then every regular space $X$ satisfying

$$
\Delta(X) \geq \kappa \geq \widehat{\mathrm{e}}(X)
$$

is 2-resolvable.

Proof. Using theorem 3.1 and theorem 2.1 it clearly suffices to show that any regular space $X$ with

$$
|X|=\Delta(X)=\widehat{\mathrm{e}}(X)=\kappa
$$

has a 2-resolvable subspace.

If there is $R \in \mathrm{RC}^{+}(X)$ with $\mathrm{e}(R)<\kappa$ then $R$ is $\omega$-resolvable by theorem 3.1, hence we may assume that $\widehat{\mathrm{e}}(R)=\kappa$ for all $R \in \mathrm{RC}^{+}(X)$. Also, if some $G \in \tau^{+}(X)$ has a dense subset $Y$ of cardinality $<\kappa$ then $\Delta(G)=\Delta(X)=\kappa$ implies that $G \backslash Y$ is also dense in $G$, hence $G$ is 2-resolvable. Thus we may also assume that every set $Y \in[X]^{<\kappa}$ is nowhere dense in $X$.

By $\operatorname{cf}(\kappa)=\omega$ we can fix a strictly increasing sequence of cardinals $\left\langle\kappa_{n}: n<\omega\right\rangle$ with $\kappa=\sum\left\{\kappa_{n}: n<\omega\right\}$. Then we may choose a sequence of sets $\left\{Y_{n}: n<\omega\right\} \subset[X]^{<\kappa}$ with $\left|Y_{n}\right|=\kappa_{n}$ and $\bigcup_{n \in \omega} Y_{n}=X$. Each $\overline{Y_{n}}$ is nowhere dense, hence we may define by a straightforward induction a sequence $\left\{U_{n}: n<\omega\right\} \subset \tau^{+}(X)$ such that $\overline{U_{n+1}} \varsubsetneqq U_{n}$ for all $n<\omega$, moreover $\bigcap\left\{U_{n}: n<\omega\right\}=\emptyset$.

Then $R_{n}=\overline{U_{n}} \backslash \operatorname{Int}\left(\overline{U_{n+1}}\right) \in \mathrm{RC}^{+}(X)$ and $\left\{R_{2 n}: n<\omega\right\}$ is clearly a discrete collection in $X$. But $\widehat{\mathrm{e}}\left(R_{2 n}\right)=\kappa$ implies the existence of a set $D_{n} \in\left[R_{2 n}\right]^{\kappa_{n}}$ that is closed discrete in $R_{2 n}$ and hence in $X$. Consequently,

$$
D=\bigcup\left\{D_{n}: n<\omega\right\} \in[X]^{\kappa}
$$

is also closed discrete in $X$, contradicting $\widehat{\mathrm{e}}(X)=\kappa$ and completing the proof.

\section{StePPing-UP RESOlVABILITY}

Let $\kappa$ be an infinite cardinal and denote by $\mathcal{L}_{\kappa}$ the class of all regular spaces $X$ that are $\kappa^{+}$-compact and satisfy $|X|=\Delta(X)=\kappa^{+}$. (We recall that the $\kappa^{+}$-compactness of $X$ means that $A^{\circ} \neq \emptyset$ for each $A \in$ $[X]^{\kappa^{+}}$.) Our aim is then to prove the following stepping up result.

Theorem 4.1. If every member of $\mathcal{L}_{\kappa}$ is $\kappa$-resolvable then actually every member of $\mathcal{L}_{\kappa}$ is $\kappa^{+}$-resolvable.

Before giving the proof of this, however, we have to formulate and prove the following lemma. 
Lemma 4.2. Assume that $X \in \mathcal{L}_{\kappa}$ has no $\kappa^{+}$-approachable subset. Then

(i) for any $A \in[X]^{\kappa^{+}}$we have $A^{\circ} \in \mathcal{L}_{\kappa}$;

(ii) there are $\kappa^{+}$many pairwise disjoint sets of the form $A^{\circ}$ with $A \in$ $[X]^{\kappa^{+}}$.

Proof. (i) It is immediate from part (2) of lemma 2.6. applied to $\kappa^{+}$ instead of $\kappa$, that for any $A \in[X]^{\kappa^{+}}$we have $\left|A^{\circ}\right|=\kappa^{+}$. Also, as $A^{\circ}$ is closed in $X$, it is $\kappa^{+}$-compact. So, to prove $A^{\circ} \in \mathcal{L}_{\kappa}$ it only remains to show that $\Delta\left(A^{\circ}\right)=\kappa^{+}$.

To see this, assume that $U$ is open with $U \cap A^{\circ} \neq \emptyset$, say $x \in U \cap A^{\circ}$. By the regularity of $X$ the point $x$ has an open neighbourhood $V$ such that $\bar{V} \subset U$. Then $x \in A^{\circ}$ implies $|V \cap A|=\kappa^{+}$, hence $\left|(V \cap A)^{\circ}\right|=\kappa^{+}$. This, in turn, implies $\left|U \cap A^{\circ}\right|=\kappa^{+}$because $(V \cap A)^{\circ} \subset \bar{V} \cap A^{\circ} \subset U \cap A^{\circ}$.

(ii) Let us note first of all that if we have $A, B \in[X]^{\kappa^{+}}$with $A^{\circ} \backslash B^{\circ} \neq$ $\emptyset$ then there is a set $C \in[A]^{\kappa^{+}}$such that $C^{\circ} \cap B^{\circ}=\emptyset$. Indeed, if $x \in A^{\circ} \backslash B^{\circ}$ and $V$ is an open neighbourhood of $x$ with $|\bar{V} \cap B| \leq \kappa$ then the choice $C=V \cap A$ clearly works.

Now we distinguish two cases.

\section{Case 1:}

There is a disjoint family $\left\{X_{\xi}: \xi<\kappa^{+}\right\} \subset[X]^{\kappa^{+}}$such that the increasing $\kappa^{+}$-sequence $\left\{Y_{\xi}^{\circ}: 0<\xi<\kappa^{+}\right\}$, where $Y_{\xi}=\bigcup_{\eta<\xi} X_{\eta}$, does not stabilize. This means that the set $I=\left\{\xi<\kappa^{+}: Y_{\xi+1}^{\circ} \backslash Y_{\xi}^{\circ} \neq \emptyset\right\}$ has cardinality $\kappa^{+}$. By our above remark then for each $\xi \in I$ there is a set $C_{\xi} \in\left[Y_{\xi+1}\right]^{\kappa^{+}}$such that $C_{\xi}^{\circ} \cap Y_{\xi}^{\circ}=\emptyset$. But this means that the members of the family $\left\{C_{\xi}^{\circ}: \xi \in I\right\}$ are pairwise disjoint, and we are done.

\section{Case 2:}

For every disjoint family $\left\{X_{\xi}: \xi<\kappa^{+}\right\} \subset[X]^{\kappa^{+}}$the sequence $\left\{Y_{\xi}^{\circ}\right.$ : $\left.\xi<\kappa^{+}\right\}$, as defined above, does stabilize.

Let us then fix a disjoint family $\left\{X_{\xi}: \xi<\kappa^{+}\right\} \subset[X]^{\kappa^{+}}$and for any $\xi<\eta<\kappa^{+}$put $Y_{\xi, \eta}=\bigcup\left\{X_{i}: \xi \leq i<\eta\right\}$. But now for each fixed $\xi<\kappa^{+}$the sequence

$$
\left\{\left(Y_{\xi, \eta}\right)^{\circ}: \xi<\eta<\kappa^{+}\right\}
$$

stabilizes, i.e. there is an $\eta(\xi)<\kappa^{+}$such that

$$
\left(Y_{\xi, \zeta}\right)^{\circ}=\left(Y_{\xi, \eta(\xi)}\right)^{\circ}=F_{\xi}
$$

whenever $\eta(\xi) \leq \zeta<\kappa^{+}$.

The sequence $\left\{F_{\xi}: \xi<\kappa^{+}\right\}$is clearly decreasing and we claim that it cannot stabilize. Indeed, assume on the contrary that there is some 
$\xi_{0}<\kappa^{+}$such that $F_{\xi}=F_{\xi_{0}}$ for all $\xi_{0}<\xi<\kappa^{+}$. We may then select a set $I \in\left[\kappa^{+} \backslash \xi_{0}\right]^{\kappa^{+}}$such that $\eta(\xi)<\zeta$ holds whenever $\{\xi, \zeta\} \in[I]^{2}$ and $\xi<\zeta$. But then the disjoint collection $\left\{Y_{\xi, \eta(\xi)}: \xi \in I\right\} \subset[X]^{\kappa^{+}}$ would witness that the set $F_{\xi_{0}}$ is $\kappa^{+}$-approachable in $X$, contradicting our assumption.

Consequently, the set $J=\left\{\xi<\kappa^{+}: F_{\xi} \backslash F_{\xi+1} \neq \emptyset\right\}$ has cardinality $\kappa^{+}$and by our introductory remark for each $\xi \in J$ there is a set $C_{\xi} \in$ $\left[Y_{\xi, \eta(\xi)}\right]^{\kappa^{+}}$such that $C_{\xi}^{\circ} \cap F_{\xi+1}=\emptyset$. But we also have $C_{\xi}^{\circ} \subset F_{\xi}$ for each $\xi \in J$, consequently the sets $\left\{C_{\xi}^{\circ}: \xi \in J\right\}$ are pairwise disjoint, completing the proof of (ii).

Proof of theorem 4.1. Let us assume, to begin with, that every member of $\mathcal{L}_{\kappa}$ is $\kappa$-resolvable. Our aim is to show that then every member of $\mathcal{L}_{\kappa}$ is $\kappa^{+}$-resolvable. Since $\mathcal{L}_{\kappa}$ is regular closed hereditary, by corollary 2.2 it suffices to prove that every member of $\mathcal{L}_{\kappa}$ has a $\kappa^{+}$-resolvable subspace.

Now, if $X \in \mathcal{L}_{\kappa}$ is such that its $\kappa^{+}$-approachable subsets form a $\pi$ network in $X$ then it follows from theorem 2.7 that $X$ is $\kappa^{+}$-resolvable. Therefore, it will suffice to show that any space $X \in \mathcal{L}_{\kappa}$ that has no $\kappa^{+}$-approachable subset contains a $\kappa^{+}$-resolvable subspace.

To see this, we may apply lemma 4.2 to obtain a family

$$
\left\{C_{\alpha}: \alpha<\kappa^{+}\right\} \subset[X]^{\kappa^{+}}
$$

such that the sets $A_{\alpha}=C_{\alpha}^{\circ}$ are pairwise disjoint. Since each $A_{\alpha} \in \mathcal{L}_{\kappa}$ is $\kappa$-resolvable by our assumption, it follows that the closed set

$$
F=\bigcap_{\alpha<\kappa^{+}} \overline{\bigcup\left\{A_{\beta}: \beta \in \kappa^{+} \backslash \alpha\right\}}
$$

is $\kappa$-nice in the sense of definition 2.14.

We claim that $F \in \mathcal{L}_{\kappa}$ holds also and this will follow if we can show that $\Delta(F)=\kappa^{+}$. To see this, let $U$ be open with $U \cap F \neq \emptyset$ and pick $x \in U \cap F$. By the regularity of $X$ the point $x$ has an open neighbourhood $V$ such that $\bar{V} \subset U$. Clearly, then

$$
I=\left\{\alpha: V \cap A_{\alpha} \neq \emptyset\right\} \in\left[\kappa^{+}\right]^{\kappa^{+}} .
$$

Let us pick for each $\alpha \in I$ a point $x_{\alpha} \in V \cap A_{\alpha}$ and consider the set $B=\left\{x_{\alpha}: \alpha \in I\right\}$. Then $B \in[X]^{\kappa^{+}}$and $B^{\circ} \subset \bar{V} \cap F \subset U \cap F$, hence $|U \cap F|=\kappa^{+}$. So we indeed have $F \in \mathcal{L}_{\kappa}$, and therefore $F$ is both $\kappa$-nice and $\kappa$-resolvable.

This argument can also be applied to any regular closed subset $R \in$ $\mathrm{RC}^{+}(X)$ to obtain a subset of $R$ that is both $\kappa$-nice and $\kappa$-resolvable. 
Thus we have concluded that the sets that are both $\kappa$-nice and $\kappa$ resolvable form a $\pi$-network in $X$. So if $\mathcal{F}$ is any maximal disjoint collection of such subsets of $X$ then corollary 2.15 can be applied to $X$ and $\mathcal{F}$ to conclude that $X$ has a $\kappa^{+}$-resolvable (open) subspace, and thus the proof is completed.

Since Lindelöf spaces are trivially $\omega_{1}$-compact, we immediately obtain from theorem 4.1 and the case $\kappa=\omega_{1}$ of theorem 3.1 the following result that was promised already in the abstract.

Corollary 4.3. Every $\omega_{1}$-compact (hence every Lindelöf) regular space $X$ with $|X|=\Delta(X)=\omega_{1}$ is $\omega_{1}$-resolvable.

We were unable to answer the natural question whether, in corollary 4.3, the assumption of $\omega_{1}$-compactness can be relaxed to the property of having countable extent.

\section{REFERENCES}

[1] El'kin A.G., On the maximal resolvability of products of topological spaces, Soviet Math. Dokl. 10 (1969), 659-662.

[2] Filatova, M. A., Resolvability of Lindelöf spaces. (Russian. English, Russian summary) Fundam. Prikl. Mat. 11 (2005), no. 5, 225-231; translation in J. Math. Sci. (N. Y.) 146 (2007), no. 1, 5603-5607

[3] Illanes, Alejandro, Finite and w-resolvability, Proc. Amer. Math. Soc. 124 (1996), no. 4, 1243-1246.

[4] I. Juhász; L. Soukup; Z. Szentmiklóssy: Resolvability of spaces having small spread or extent, Topology Appl., 154(2007) , 144-154.

[5] I. Juhász; Z. Szentmiklóssy; Interpolation of $\kappa$-compactness and PCF, Comment. Math. Univ. Carolin. 50 (2009), no. 2, 315-320.

[6] Malykhin, V.I., Borel resolvability of compact spaces and their subspaces, Math. Notes 64 (1998), No. 5, 607-615.

[7] O. Pavlov, On resolvability of topological spaces, Topology Appl. 126 (2002), no. 1-2, 37-47.

[8] E. G. Pytkeev, Resolvability of countably compact regular spaces, Proc. Steklov Inst. Math. 2002, Alg., Top., Math. Anal., suppl. 2, S152-S154.

Alfréd Rényi Institute of Mathematics, Hungarian Academy of SciENCES

E-mail address: juhasz@renyi.hu

Alfréd Rényi Institute of Mathematics, Hungarian Academy of SciENCES

E-mail address: soukup@renyi.hu

URL: http://www.renyi.hu/soukup

EÖTVÖS LORÁND UNIVERSITY OF BUDAPEST

E-mail address: zoli@renyi.hu 